\title{
Localized Edema, CTCAE
}

National Cancer Institute

\section{Source}

National Cancer Institute. Localized Edema, CT CAE. NCI Thesaurus. Code C143654.

A disorder characterized by swelling due to excessive fluid accumulation at a specific anatomic site. 